\title{
Content based Structural Recognition for Image Classification using PSO Technique and SVM
}

\author{
Abhishek Pandey \\ Dept. of CSE \\ UIT-RGPV Bhopal (M.P)
}

\author{
Anjna Jayant Deen \\ Dept. of CSE \\ UIT-RGPV Bhopal (M.P)
}

\author{
Rajeev Pandey, Ph.D \\ Dept. of CSE \\ UIT-RGPV Bhopal(M.P)
}

\begin{abstract}
The issue of SVMs parameter optimization with particle swarm optimization (pso) provide the optimum solution. This new classification approach may be an efficient alternative, in existing paradigms. PSO technique work with high dimensional datasets and mixed attribute data. The structure of the image is recognized through PSO technique which provide optimized parameter for SVM. This approach determines the performance of image classification after structural recognition based on content of image and comparing the obtained results with those reported for various other classification approaches.

PSO-SVM technique can be applied mixed-attribute, hyperspectral data, hyperdimension spaces \& problem description spaces and it can also be a competitive alternative to well established classification techniques. The optimized process of data reduces the unclassified region of support vector machine and improves the performance of image classification. The feature of region of image is classified by PSO-SVM technique in inside the image. Cassified features are increase recogniztion ratio because the feature of image is optimized.
\end{abstract}

\section{General Terms}

Pattern Recognition, high dimensional image classification et. al.

\section{Keywords}

Structural recognition, PSO technique, SVM classifier, image classification.

\section{INTRODUCTION}

Nowadays, Technology in digital image processing is emerging rapidly. So, the accuracy and performance of images in the field of image recognition, image segmentation, image reconstruction \& image classification plays a vital role in digital image processing. My research work in Content based image recognition then classification of images are a key part of digital image processing. The enlargement in the computer technologies and the advent of the Nation wise Wide Web, there has been an explosion in the amount and complexity of digital data being generated, stored, transmitted, analyzed, and accessed. In order to make use of this vast amount of data, efficient and effective techniques to retrieve multimedia information based on its content need to be developed. Image recognition is challenging $\&$ new emerging field of image processing. This application importance in image analysis and design field. The image content is color, texture and shape[11].

The major image feature is the structure of the object contained in the image Shape feature of the image may be defined as the characteristic surface configuration of an object; an outline or contour[18]. Shape representations can be generally divided into two categories:

- Boundary-based, and

- Region-based.
The shape of an object is a binary image representing the extent of objects. In region-based considers the shape being composed of a set of two-dimensional regions, while the boundary based representation presents the shape of its outline. Region-based feature vectors often result in shorter feature vectors and simpler matching algorithms. Object region features are recognized on the basis of pixel values in digital image processing.

Recently evolutionary algorithms are used for optimization \& Recognition:

1. Genetic algorithm (GA).

2. Particle swarm optimization (PSO)

3. Ant colony optimization (ACO)

4. Simulated annealing algorithm (SA)

This Algorithm has been employed to optimize the SVMs parameters for their better global search abilities against numerical optimization methods[23]. PSO algorithm is responsible for optimum solution for parameters selection. Particle Swarm Optimization (PSO) algorithm is a global optimization method originally [1] [2]. It is swarm intelligence algorithm that emulates swarm behaviors such as birds flocking and fish schooling[3]. It is a population-based iterative learning algorithm that shares some common characteristics with other evolutionary computation (EC) algorithms [25]. The PSO algorithm works with noisy images. This algorithm efficiently work with edge detection, line detection and point detection[24].

For the classification of lower content of the image we used support vector machine. Support vector machine is a linear and bilinear classifier [5]. Machine Learning is considered as a subfield of Artificial Intelligence and it is concerned with the development of techniques and methods which enable the computer to learn. Image recognition is a basic step of Machine learning.

Special attention is given to two major potential problems encountered in the classification domain: datasets with high dimensionality and mixed attribute with noisy data[20]. Particle Swarm Optimisation (PSO) is a population-based meta-heuristic method for solving global and local optimisation problems based on social-psychological principles[22]. Compared with some heuristic methods such as genetic algorithms, the most important general advantages of PSO are ease of its implementation, memory for each particle and high speed of convergence[25].

\section{RELATED WORK}

Particle Swarm Optimization (PSO) algorithm is a global optimization method originally. It is swarm intelligence algorithm that emulates swarm behaviors such as birds flocking and fish schooling. It is a population-based iterative learning algorithm that shares some common characteristics with other evolutionary computation (EC) algorithms. ACO makes probabilistic decision in terms of the artificial pheromone trails and the local heuristic information. This allows ACO to explore 
larger number of solutions than greedy heuristics. Another characteristic of the ACO algorithm is the pheromone trail evaporation, which is a process that leads to decreasing the pheromone trail intensity over time. It is suggested by Qin Da et al[13].

Addressing the issue of SVMs parameters optimization, this study proposes an efficient memetic algorithm based on particle swarm optimization algorithm (PSO) and pattern search (PS). In the proposed memetic algorithm, PSO is responsible for exploration of the search space and the detection of the potential regions with optimum solutions, while pattern search (PS) is used to produce an effective exploitation on the potential regions obtained by PSO.This is worked by Yukun Bao et al[22]. Support vector machine (SVM) is the state-of-the-art classification method, and the doubly regularized SVM (DrSVM) is an important extension based on the elastic net penalty. DrSVM has been successfully applied in handling variable selection while retaining (or discarding) correlated variables. However, it is challenging to solve this model.This is suggested by Dehua Liu[25]. This study initially aims at optimizing the accuracy of SVM classifier by detecting the subset of best informative features and estimating the best values for regularization of kernel parameters for SVM model[16]. In order to achieve this PSO based optimized framework is used. PSO-SVM algorithm combines two machine learning methods by optimizing the parameters of SVM using PSO with fast feature extraction metod. PSO starts with n-randomly selected particles and searches for the optimal particle iteratively. Each particle is a m-dimensional vector and represents a candidate solution. SVM classifier is built for each candidate solution to evaluate its performance through the cross validation method. PSO algorithm guides the selection of potential subsets that lead to best prediction accuracy[16]. The algorithm uses the fit particles to contribute to the next generation of n-candidate particles. Thus, on the average, each successive population of candidate particles fits better than its predecessor. This work is Fatima Ardjani et al[16].

\subsection{PSO Technique}

PSO was first described by James Kennedy and Russell Eberhart in 1995. This algorithm apply in noisy feature of images. This algorithm is swarm intelligence based. The observatzion of swarming habits of animals such as birds or fish. The PSO algorithm maintains multiple potential solutions at one time. During each iteration of the algorithm, each solution is evaluated by an objective function. Each solution is represented by a particle in the fitness landscape (search space).The particles "fly" or "swarm" through the search space to find the maximum value returned by objective function

The PSO algorithm consists of just three steps:

1. Evaluate the fitness of each particle.

2. Update individual and global best.

3. Update velocity and position of each particle.

These steps are repeated until some stopping condition is met. Performance of particle is fitness of each particle. (local best value is the fitness value of particle). Each particle's velocity is updated using this equation:

$\operatorname{vi}(t+1)=\operatorname{wvi}(i)+c_{1} r_{1}[\hat{x} \mathrm{i}(t)-x i(t)]+c_{2} \mathrm{r}_{2}[g(t)-x i(t)] \ldots \ldots \ldots(1)$

where,

$i$ is the particle index.

$w$ is the inertial coefficient.

$c 1, c 2$ are acceleration coefficients.

$r 1, r 2$ are random values

Regenerated every velocity update particle.

$\operatorname{vi}(t)$ is the particle's velocity at time $\mathrm{t}$. $\mathrm{xi}(t)$ is the particle's position at time $\mathrm{t}$.

'xi $(t)$ is the particle's individual best solution as of time t.

$\mathrm{g}(t)$ is the swarm's best solution as of time $\mathrm{t}$.

Each particle's position is updated using this equation:

$\mathrm{xi}(t+1)=\mathrm{xi}(t)+\mathrm{vi}(t+1) \ldots \ldots \ldots \ldots \ldots \ldots(2)$

Particle Swarm Optimization (PSO) algorithm is a global optimization method originally [1][2]. It is swarm intelligence algorithm that emulates swarm behaviors such as birds flocking and fish schooling[8]. It is a population-based iterative learning algorithm that shares some common characteristics with other evolutionary computation (EC) algorithms [3]. However, particle flying in the search space and adjusting its flying trajectory according to its personal best experience and its neighborhood's best experience rather than through particles undergoing genetic operations like selection, crossover, and mutation [4]. Owing to its simple concept and high efficiency, PSO has become a widely adopted optimization technique and has been successfully applied to many real-world problems [8]. PSO searches for an optimum through each Particle swarm optimization (PSO) relies on its learning strategy to guide its search direction. Traditionally, each particle utilizes its historical best experience and its neighborhood's best experience through linear summation. Such a learning strategy is easy to use.

\subsection{Support Vector Machine}

Support vector machine is a linear and bilinear classifier[18] Support Vector Machine (SVM) was first heard in 1992, introduced by Bose, Guyon, and Vapnik in COLT-92. Support vector machines (SVMs) are a set of related supervised learning methods used for classification and regression [8]. They belong to a family of generalized linear classifiers. In other terms, Support Vector Machine (SVM) is a classification and regression prediction tool that uses machine learning theory to maximize predictive accuracy while automatically avoiding over-fit to the data. Support Vector machines can be defined as systems which use the hypothesis space of a linear function in a high dimensional feature space, trained with a learning algorithm from optimization theory that implements a learning bias derived from statistical learning theory[25]. It is also being used for many applications, such as handwriting analysis, phase analysis and so forth, especially for pattern classification and regression based applications. The foundations of Support Vector Machines (SVM) have been developed by Vapnik and gained popularity due to many promising features such as better empirical performance.

SVM is a novel neural network technique, which can be applied in classification and regression analysis. Consider a training data set $\left\{\left(\mathrm{x}_{\mathrm{i}}, \mathrm{y}_{\mathrm{i}}\right)\right\}_{\mathrm{i}=1}^{\mathrm{n}}$ where $x_{i}$ presents the input vector and $y_{i} \varepsilon\{1,-1\}$ presents the class label. Determination of optimum hyper plane can be used to solve the following optimization problem:

Min

$$
\frac{1}{2}\|w\|^{2}
$$

Subject to

$$
\mathrm{y}_{\mathrm{i}}\left(\mathrm{w} \cdot \mathrm{x}_{\mathrm{i}}+\mathrm{b}\right) \geq 1
$$

In order to solve the above optimization problem, two positive slack variables are introduced. Then, the optimization problem is given as follows:

Min

$$
\begin{gathered}
\frac{1}{2}\|w\|^{2}+C \sum_{i=1}^{n} \xi \ldots \ldots \ldots \ldots \ldots \ldots \ldots(4) \\
\mathrm{y}_{\mathrm{i}}\left(\mathrm{w} . \mathrm{x}_{\mathrm{i}}+\mathrm{b}\right) \geq 1-\xi_{\mathrm{i}}, \xi_{\mathrm{i}} \geq 0
\end{gathered}
$$

where $\xi_{\mathrm{i}} \xi_{\mathrm{i}}$ are the slack variables; $C$ is a regularization constant. Finally, the classification function is written as follows: 


$$
\left.f(x)=\operatorname{sign}\left(\sum_{i=1}^{n} \alpha_{i} y_{i} k\left(x_{i}, x_{j}\right)\right)+b\right)
$$

where $\alpha_{i} \square$ is the Lagrange multipliers.

\section{PROPOSED METHODOLOGY}

This research work aims at providing more accurate result compare to other techniques. Proposed methodology used following major steps:

I. Image Preprocessing- The dataset of images in standard size through crops of images. Images are in normalized images using preprocessing techniques.

II. Feature Extraction- Extraction of features of images are based on content based image retrieval (CBIR) technique. A typical CBIR system automatically extracts visual attributes (color, shape, texture and spatial information) of each image in the database based on its pixel values and stores them into a different database within the system called feature database $[6,11]$. The feature data for each of the visual attributes of each image is very much smaller in size compared to the image data.

III. PSO-SVM Technique-Proposed PSO-SVM system of classification. This study initially aims at optimizing the accuracy of the SVM classifier by detecting the subset of best informative features and estimating the best values for regularization of kernel parameters for SVM model.The PSOSVM with fast texture extraction feature is apply for classification. In order to achieve this PSO based optimized framework is used. PSO-SVM algorithm combines two machine learning methods of optimizing the parameters of SVM using PSO [23]. PSO starts with n-randomly selected particles and searches for the optimal particle iteratively. Each particle is a mdimensional vector and represents a candidate solution. SVM classifier is built for each candidate solution to evaluate its performance through the cross validation method[9].The algorithm uses the most fit particles to contribute to the next generation of $n$-candidate particles. Thus, on the average, each successive population of candidate particles fits better than its predecessor. This process continues until the performance of SVM converges. PSO is used to find optimal feature subsets by discovering the best feature combinations as they fly within the problem space from the processed data sets[23].

The procedure is described proposed PSO-SVM approach is as follows.

1. Initializing PSO with population size, inertia weight and generations without improving.

2. Evaluating the fitness of each particle.

3. Comparing the fitness values and determines the local best and global best particle.

4. Updating the velocity and position of each particle till the value of the fitness function converges.

5. After converging, the global best particle in the swarm is fed to the SVM classifier for training.

6. Training the SVM classifier. The PSO-SVM takes the advantage of minimum structural risk of SVM and the quick global optimizing ability of PSO.

The application of the algorithm of optimization by particulate swarm, like any evolutionary algorithm, is influenced by factors such as the criterion of the stop, the structure of the particle, the objective function.

We testify the classification performance of the proposed method. The selection of the parameters of support vector machine will critically affect its results. The image used to testify the classification performance of the proposed method.
The SVM, BP neural network and RBF neural network are used to compare with PSO-SVM with fast texture feature extraction method. The correct rate is used to measure the classification performance of PSO-SVM. The classification accuracy of PSOSVM.

Correct rate $=\frac{\text { Correct number }}{\text { Correct number }+ \text { error number }}$

If the error number is less and correct number is more then the correct rate of classification is greater then the percentage of recognition will be increased.

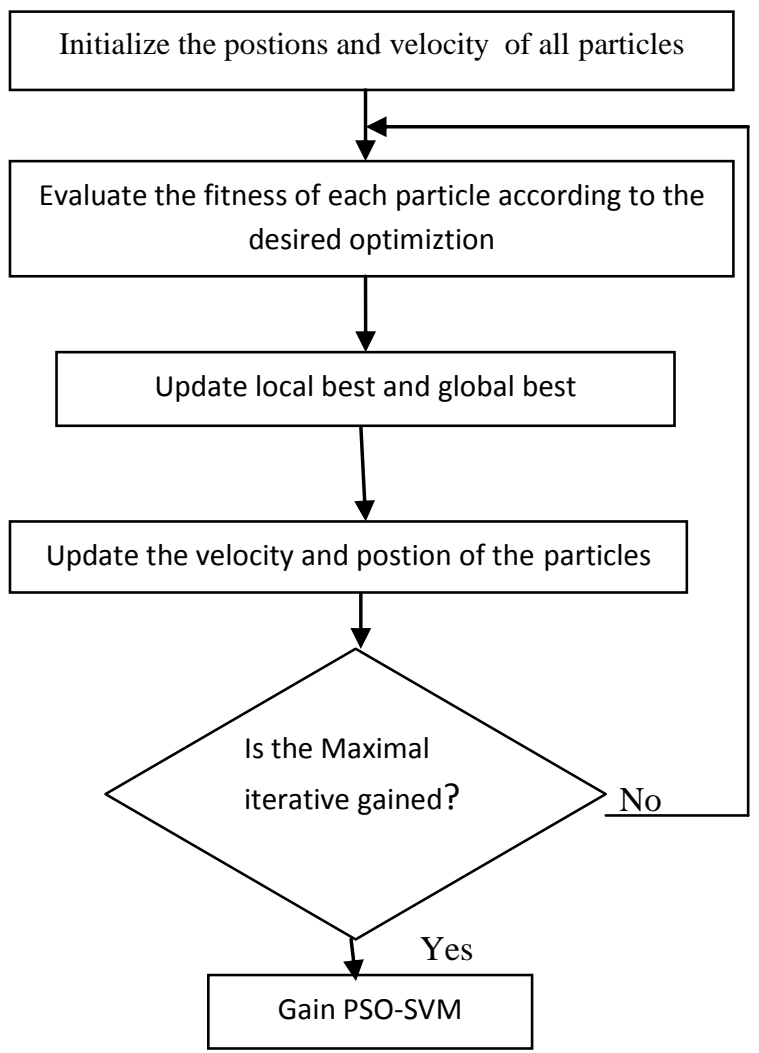

Figure 1: The process of support vector machine trained by particle swarm optimization

\section{RESULT ANALYSIS}

The classification is tested on satellite, remote sensing and Land Sat images. These are images taken by the satellite. Here original image is taken first as input then the region feature extracted, then its interest is selected for training on the bases of region. 


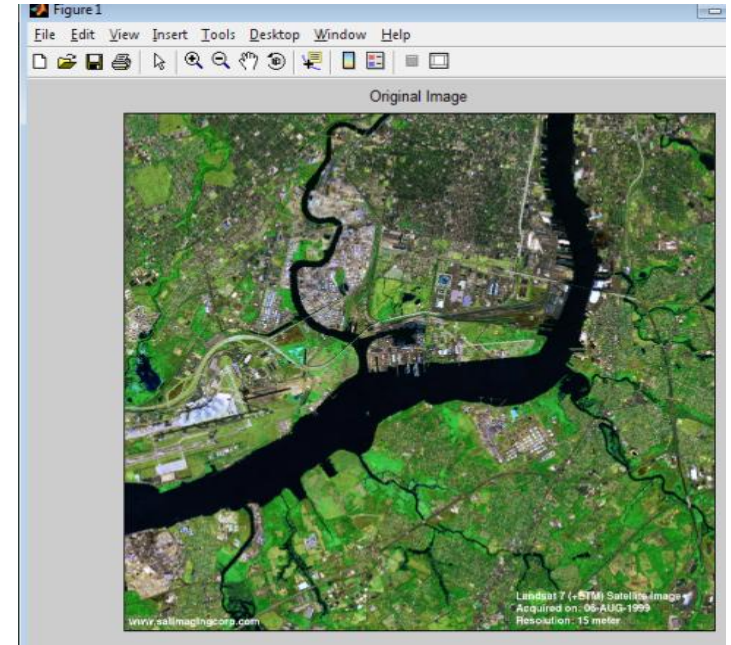

Figure 2: Satellite image1

Here figure 1 shows that the main satellite image, which is read first, and in the next step the input query image is proceed for the selected region of interest with accordingly similar classified image.

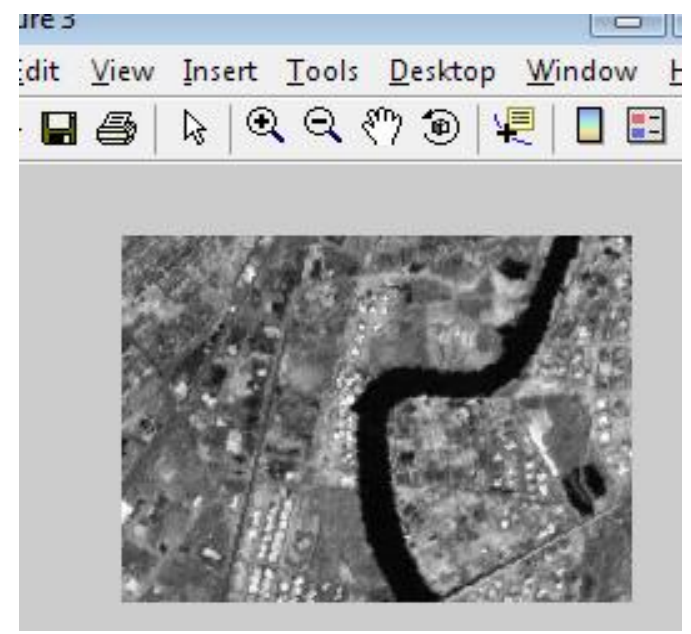

Figure 3: Region of Interest

Now here figure 2 shows that the selected region of interest of the input taken image before. And figure 3 shows that the highlighted region of the image taken for testing.

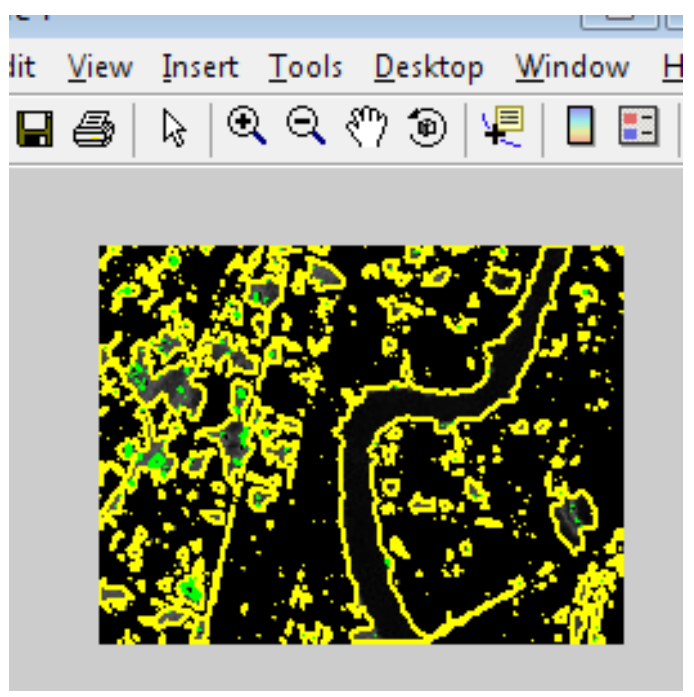

Figure 4: Region based classified image

Here table 1 shows that the accuracy, kappa coefficient value of the satellite and Land Sat image on the basis of confusion Matrix. The analysis is done on the basis of existing work. The existing technique is being trained using SVM training and hence the accuracy is less. In the table shows the accuracies of different image taken for the testing. Kappa value represent the categorical value of features. Kappa coefficient (k), which is believed to be a better representation of the general quality of an image classification, because it removes the effects caused by differences in sample size and accounts for the off-diagonal elements in the error matrix. It also allows different classifications to be compared statistically.

Table 1: Accuracy comparison of SVM and PSOSVM

\begin{tabular}{|c|c|c|}
\hline kappa value & With SVM & With PSOSVM \\
\hline 0.4525 & 81.6551 & 89.5652 \\
\hline 0.4752 & 82.5475 & 87.5641 \\
\hline 0.3954 & 81.6594 & 85.6621 \\
\hline 0.4215 & 82.5475 & 88.2245 \\
\hline 0.4712 & 82.7594 & 91.6583 \\
\hline 0.3953 & 86.6547 & 90.5264 \\
\hline 0.5445 & 84.3162 & 89.4545 \\
\hline
\end{tabular}

As shown in the figure 4 is the result analysis and comparison of the SVM method and PSOSVM proposed method. The analysis shows the performance of the proposed PSOSVM, the proposed technique has more accuracy as compared to the only SVM method. 


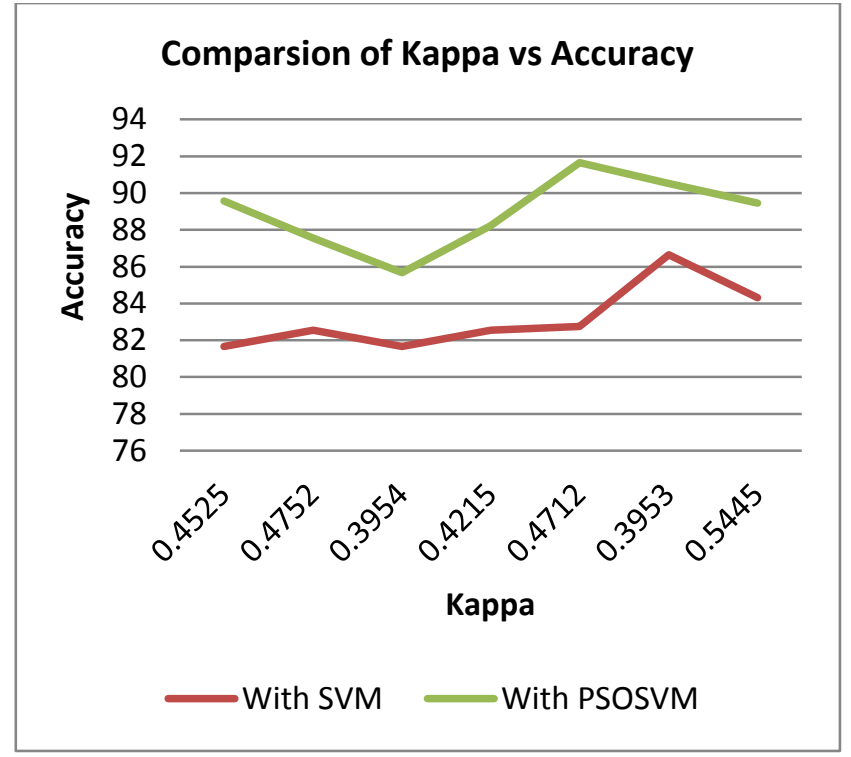

\section{Figure 5: Comparison of SVM technique with Proposed PSOSVM method}

Recognition rate of image is much more to other techniques with PSOSVM because classification accuracy is enhanced.

Results are described with other techniques-

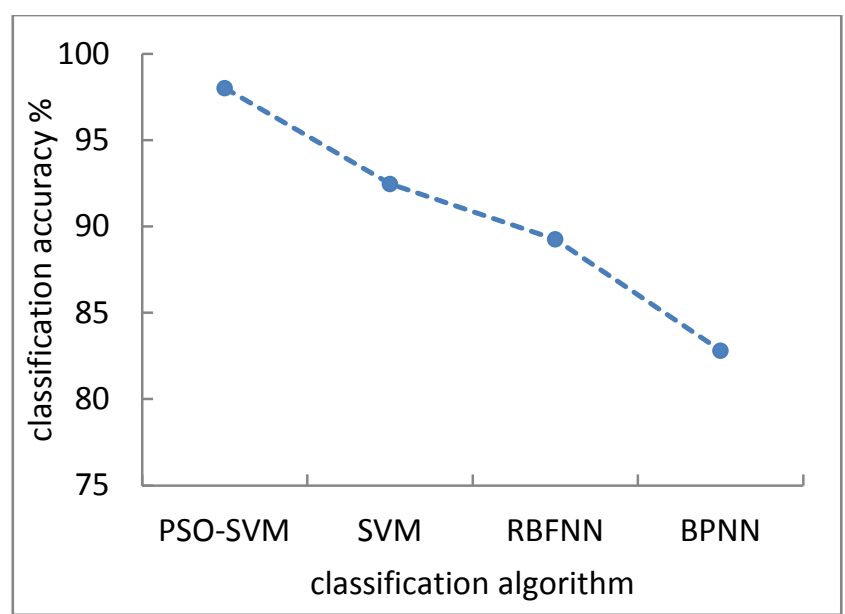

Figure 6: The classification accuracy of PSO-SVM compared with SVM, BP neural network, RBF neural network.

- Due to randomized aspects of PSO algorithm, the optimization process would need to be run several times to determine if results are consistent.

- Alternative PSO parameters can be attempted, and their effectiveness measured.

\section{CONCLUSION}

Significant speedup using PSO over exhaustive search. This technique provides the optimum solution efficiently for the image classification after image recognition as compare to other techniques. Another alternative can be used as pso technique. In my research study, recognition of images with pso techniques for classification using svm provide better result as compared to other techniques (such as ACO optimization, neural network and radial basis network). PSO algorithm with SVM working with efficiently and recognition performance is is increased compare to other techniques. PSO Algorithm's modification or alternative will be apply in the future.

\section{FUTURE WORK}

The Limitation of the PSO approach when the number of classes is important in multiclass databases. From the reported classification performances for various datasets, the results clearly indicate that PSO out performed other classification methods in accuracy. Nevertheless, the time con- sumption of the training stage is substantially higher than with other techniques. The other alternative can be used in which time consumption problem would be removed.

\section{REFERENCES}

[1]. J. Kennedy and R. C. Eberhart, "Particle swarm optimization," in Proc. IEEE Int. Conf. Neural Netw., vol. 4, pp. 1942-1948, 1995.

[2]. R. C. Eberhart and J. Kennedy, "A new optimizer using particle swarm theory," in Proc. $6^{\text {th }}$ Int. Symp. Micromach. Human Sci., pp. 39-43, 1995.

[3]. Y. H. Shi and R. C. Eberhart, "Comparison between genetic algorithms and particle swarm optimization" in Proc. 7th Int. Conf. Evol. Program, LNCS 1447, pp. 611-616, 1998

[4]. Q. Zhang, E. Izquierdo, A new approach to image retrieval in a multi-feature space, in: International Workshop on Image Analysis for Multimedia Interactive Services, 2006.

[5]. Jianxin Zhou, Ke Gao and Jintao Li." An effective method in Relevance Feedback with SVM. Journal of ComputerAided Design\& Computer Graphics", Vol.19, No.4, 2007.

[6]. Y. D. Chun, N. C. Kim, and I. H. Jang, "Content-based image retrieval using multiresolution color and texture features," IEEE Trans. Multimedia, vol. 10, no. 6, pp. 1073-1084, Oct. 2008.

[7]. D. N. F. Awang iskandar james a. Thom s. M. M. Tahaghoghi "content-based image retrieval using image regions as query examples" in Australian computer society 2008 .

[8]. Farid Melgani, Yakoub Bazi "Classification of Electrocardiogram Signals with Support Vector Machines and Particle Swarm Optimization" IEEE Transactions On Information Technology In Biomedicine, Vol. 12, No. 5, September 2008.

[9]. S. Osowski, R. Siroic, T. Markiewicz, and K. Siwek, "Application of support vector machine and genetic algorithm for improved blood cell recognition," IEEE Trans. Instrum. Meas., vol. 58, no. 7, pp. 2159-2168, Jul. 2009.

[10]. P. Schnitzspan, M. Fritz, S. Roth, B. Schiele, Discriminative Structure Learning of Hierarchical Representations for Object Detection, Computer Vision and Pattern Recognition, IEEE Computer Society Conference $\begin{array}{llll}\text { on } & 0 & \text { (2009) }\end{array}$ doi:http://doi.ieeecomputersociety.org/10.1109/CVPRW.20 09.5206544 .

[11]. C.H. Lin, R.T. Chen and Y.K. Chan, "A smart contentbased image retrieval system based on color and texture feature", Image and Vision Computing vol.27, pp.658-665, 2009.

[12]. Andrea Paoli, Farid Melgani, Edoardo Pasolli "Clustering of Hyperspectral Images Based on Multiobjective Particle 
Swarm Optimization" IEEE Transactions On Geoscience And Remote Sensing, Vol. 47, No. 12, December 2009

[13]. Qin Da “ A Hybrid PSO/ACO Algorithm for Land Cover Classification" in IEEE conference 2010.Heng chen1, zhicheng zhaol 'an effective relevance feedback algorithm for image retrieval” 978-1-4244-6853-9/10/ 2010 IEEE.

[14]. LI Linyi and LI Deren "Fuzzy Classification of Remote Sensing Images Based on particle Swarm Optimization" in International Conference on Electrical and Control Engineering in 2010.

[15]. Shuaishi Liu; Yantao Tian; Cheng Peng; Jinsong Li; "Facial expression recognition approach based on least squares support vector machine with improved particle swarm optimization algorithm "IEEE International Conference on Robotics and Biomimetics (ROBIO), 2010 pp: $399-404$

[16]. Fatima Ardjani, Kaddour Sadouni "Optimization of SVM Multiclass by Particle Swarm (PSO-SVM)" I.J.Modern Education and Computer Science, 2010, 2, 32-38 Published Online December 2010 in MECS (http://www.mecspress.org/).

[17]. He Yang, Qian Du "Particle swarm optimization-based dimensionality reduction for hyperspectral image classification", Geoscience and Remote Sensing Symposium (IGARSS), 2011 IEEE International , 24-29 July 2011, ISSN : 2153-6996 Page(s): $2357-2360$.

[18]. Siu-Yeung Cho. "Content based structural reconition for flower image classification." 2011 IEEE .Division of engineering. The university of nottingm, china.

[19]. Manimala Singha and K. Hemachandran: "Content Based Image Retrieval using Color and Texture", Signal \& Image Processing : An International Journal (SIPIJ) Vol.3, No.1, February 2012

[20]. Yu ZENG a, Jixian ZHANG a, J.L. van GENDEREN b, Guangliang WANG a, "SVM-based Multi-textural Image Classification and Its Uncertainty Analysis." Chinese Academy of Surveying and Mapping, Beijing 100830, P.R.China; b University of Twente, 2012 International Conference on Industrial Control and Electronics Engineering.

[21]. Felci Rajam, S. Valli :" A Survey on Content Based Image Retrieval” Life Sci J 2013; 10(2): 2475- 2487]. (ISSN: 1097-8135). http://www.lifesciencesite.com 343.

[22]. Yukun Bao n, ZhongyiHu,TaoXiong "A PSO and pattern search based memetic algorithm for SVMs parameters optimization." 2013 Elsevier B.V, in Neurocomputing. Department of Management Science and Information
Systems, School of Management, Huazhong University of Science and Technology, Wuhan 430074, PR China.

[23]. Nabila Nouaouria a,n, MounirBoukadoum a, RobertProulx b "Particle swarm classification: A survey and positioning." 2013 Elsevier B.V. Pattern Recognition.Contents available in journal homepage: www.elsevier.com/locate/pr. Department of Computer Science, CP. 8888, Succ. Centre-ville, Montreal, QC, Canada $\mathrm{H} 3 \mathrm{C} 3 \mathrm{P} 8$.

[24]. Mahdi Setayesh , Mengjie Zhang a, Mark Johnston "A novel particle swarm optimisation approach to detecting continuous, thin and smooth edges in noisy images." 2013. Elsevier B.V. Pattern Recognition. Contents available in journal homepage: www.elsevier.com/locate/pr.

[25]. Dehua Liu,HuiQian n, GuangDai,ZhihuaZhang “ An iterative SVM approach to feature selection and classification in high-dimensional datasets." 2013. Elsevier B.V. Pattern Recognition. Contents available in journal homepage: www.elsevier.com/locate/pr. College of Computer Science \& Technology, Zhejiang University, Hangzhou 310027, China.

\section{AUTHERS' PROFILE}

Abhishek Pandey (Birth Place-Rewa(M.P.) D.O.B.01.05.1987) received his Bachelor's degree in Information technology from SATI Vidisha,(m.p.) India in 2010

At present, he is pursuing M.E. degree in Computer Science \& Engineering from UIT- RGPV, Bhopal India. His research areas are Image processing, recognition \& classification.

Prof. Anjna jayant deen (Birth Place- Bhopal, D.O.B.22.07.1973) has received her Bachelor's degree in Computer Science \& Engineering from UIT (GEC)-RGPV, Bhopal India in 1993, and completed M.Tech., degree from Maulana Azad National Institute of Technology, Bhopal, India in 2007.

At present, She is working as an Assistant Prof. in UIT-RGPV Bhopal since 2007. She has been published in various research papers in National and International Journal. Her areas of interest include Computer Networks, Neural Networks and Internet.

Dr. Rajeev Pandey received his Bachelor's degree in Computer Science \& Engineering from IET, DR. B.R.A. University Agra, U.P. India.M.E. (Computer Science \& Engineering) from Dr. B.R.A. University, Agra in 2004. Ph.D. in 2010 from Dr.B.R.A. University, Agra, U.P. India. $\mathrm{He}$ is also Pursuing Ph.D. (Computer Science \& Engineering) from RGPV, Bhopal, M.P. India. At present, he is working as an Assistant Prof. in UITRGPV, Bhopal since 2007. 